\title{
Comparative Anatomy of the Larynx in Man and the Chimpanzee: Implications for Language in Neanderthal ${ }^{1}$
}

\author{
DEAN FALK \\ Department of Anthropology, University of Michigan, Ann Arbor, \\ Michigan 48104
}

\author{
KEY WORDS Hyoid bone · Swallowing · Larynx · Speech · \\ Chimpanzee ' Neanderthal.
}

\begin{abstract}
Using the larynges of the newborn human and chimpanzee as models, Lieberman and Crelin ('71) and Lieberman, Crelin and Klatt ('72) have reconstructed the larynx of the "classic" Neanderthal La Chapelle aux Saints. The authors used their reconstructed vocal tract to generate linguistic functions which led them to conclude that Neanderthal lacked the ability to produce fully articulate human speech. In this paper, it is shown that their reconstruction of the larynx of Neanderthal is based on a placement of the hyoid bone of La Chapelle which is too high. The reconstructed hyoid bone has been placed in a position unlike that occupied by hyoid bones of newborn humans, adult humans, stillborn chimpanzees or adult chimpanzees. In any laryngeal reconstruction, the function of swallowing must be taken into account. The ability of the reconstructed Neanderthal to swallow is discussed in light of a comparative analysis of swallowing in man and the chimpanzee. It is concluded that the statement that Neanderthal was less than fully articulate remains unsubstantiated because it rests on a questionable reconstruction of the larynx.
\end{abstract}

Neanderthal man's anatomic capability for articulate speech has been the subject of investigation by Lieberman and others (Lieberman and Crelin, '71; Lieberman et al., '72). To that purpose they reconstructed the vocal tract of the Neanderthal fossil specimen from La Chapelle aux Saints, using the following procedures: (1) locating the position of the hyoid bone and thus of the larynx, (2) reconstructing the tongue and pharynx, (3) building the laryngeal, oral and pharyngeal cavities, and (4) casting the supralaryngeal air passages, or vocal tract. By computer analysis, a determination was then made of the speech sounds which the vocal tract, as reconstructed, would be capable of producing. They concluded that Neanderthal would not be able to make the vowel sounds $/ a /, / i /$, or $/ u /$ which delimit the human vowel space. He would, however, have been able to produce some vowels (such as /I/ and /e/) and some labial and dental consonants (such as $/ \mathrm{b} /$ and /d/ ). In other words, Neanderthal would not have been able to "produce the full range of human speech" (Lieberman and Crelin, '71:220).

Their reconstruction of the vocal tract of Neanderthal was patterned after vocal tracts of newborn human and adult chimpanzees. I believe, however, that they reconstructed the hyoid bone of La Chapelle in a position too high to allow swallowing. Morris ('74) has also recently criticized Lieberman and Crelin's positioning of the hyoid bone. The purpose of this paper is to demonstrate that the proper model for reconstructing the larynx of Neanderthal is an adult human rather than a newborn human and adult chimpanzee.

\section{MATERIALS AND METHODS}

The submandibular and laryngeal regions of two male chimpanzee cadavers,

1 An earlier version of this paper was read at the April, 1973 meetings of the American Association of Physical Anthropologists in Dallas, Texas. 
one juvenile and one subadult, were dissected. Particular attention was given to the laryngeal air sacs, hyoid bones and epiglottises. Both larynges were excised and photographed.

Hyoid bones of one chimpanzee, gorilla and gibbon were sketched and photographed at the Smithsonian Institute in Washington, D. C., while those of $30 \mathrm{hu}$ man adults were observed at the School of Medicine of the University of Michigan.

Original photographs of La Chapelle aux Saints were examined.

Lateral radiographs of the submandibular regions of seven female and seven male human infants and eight female and seven male human adults were observed. Lateral radiographs of one living adult, female chimpanzee (fig. 1) and two stillborn chimpanzees were also examined. These radiographs were examined in order to determine the position of the hyoid bone relative to the inferior edge of the mandible. These positions were compared with the corresponding position of the hyoid bone of the reconstructed Neanderthal.

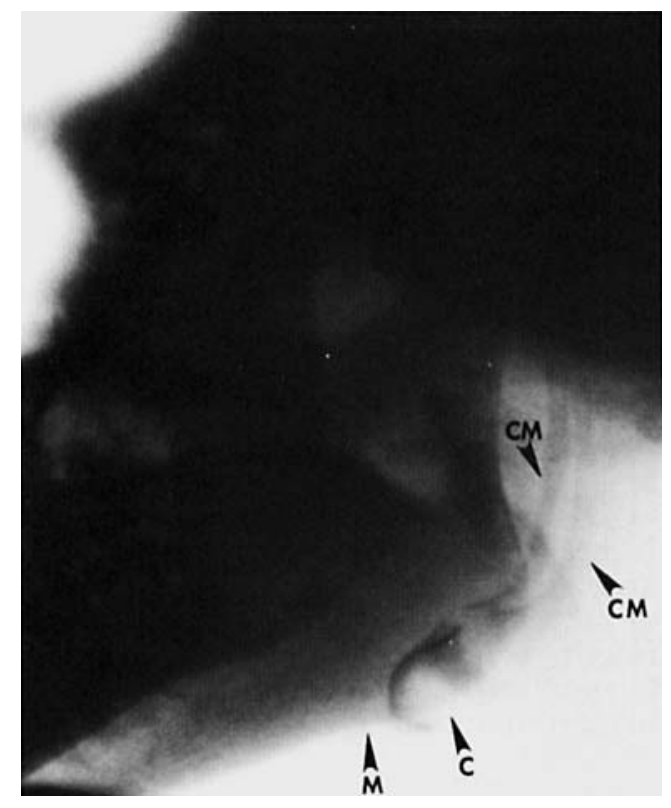

Fig. 1 Radiograph of hyoid bone relative to mandible in adult chimpanzee. Oblique view, see also figure 2 for corrected view. Abbreviations: $M$, mandible; $\mathrm{C}$, corpus of hyoid bone; CM, cornu majus (greater horn) of hyoid bone.
A transparent 8-squares-per-inch grid was superimposed on each lateral view radiograph with the grid's horizontal axis extending through gnathion and gonion and the vertical axis bisecting the gnathiongonion line. The position of the hyoid bone relative to the two axes was then recorded by marking, for each specimen, the most anterior, superior square of the grid which completely covered the corpus of the hyoid bone. In the case of human infants and stillborn chimpanzees whose hyoid bones were so small that no square of the grid completely covered them, the square which covered the largest portion of corpus was recorded.

In addition to the above lateral radiographs of subjects whose heads were oriented vertically, 130 radiographs of 65 adult humans in positions of head flexion and head extension were examined. The effect of head flexion and extension on position of the hyoid bone relative to the mandible was observed.

In the radiographs of the adult female chimpanzee, the anterior portion of the mandible is not visible. A cast of an adult female chimpanzee mandible was used to reconstruct the missing portion of the mandible and hence to determine the position of gnathion. The radiograph also shows the mandible in a tilted (oblique) position. The cast of the chimpanzee mandible, a cast of a hyoid bone of a chimpanzee, and a simulated hyoid bone wired to the cast of the chimpanzee mandible were used to estimate the position of the hyoid bone relative to the mandible in a true lateral view.

\section{RESULTS}

Figure 2 summarizes the results concerning relative position of the hyoid bone in chimpanzees and humans with vertically oriented heads. The corpora of the hyoid bones of all 14 human infants studied are posterior to the vertical axis and within 0.50 inch of it. Although figure 2 illustrates only the positions of the corpora, it should be noted that in the case of each infant and adult human the entire hyoid bone appeared inferior to the mandible. Although analysis based on sex revealed no sex difference in the distribution of the hyoid bone for infants, figure 2 sug- 
gests that there may be a positive correlation between the age in months and the distance below the mandible of the hyoid bone. The entire hyoid bone of each of the two stillborn chimpanzees also appeared inferior to the mandible. Figure 2 suggests that location of the hyoid bone of stillborn chimpanzees falls within the range of those of human infants.

Figure 2 shows that the anterior corpora of the hyoid bones of two adult human male specimens are located one and two squares anterior to the vertical axis. Those of all other adult human specimens fall posterior to the axis and within 0.75 inch of it. Although figure 2 reveals no sex difference in the distance of the hyoid bone inferior to the mandible, a sex difference does seem to exist relative to the anterior position of the hyoid bone, those of males being farther forward than those of females. (This is perhaps due to an "Adam's Apple Effect" caused by differential growth of the thyroid cartilage in pubescent males. )

The 130 radiographs of 65 human adults, with heads flexed in one radiograph and extended in another, showed the entire hyoid bone always inferior to the mandible in extension and in 56 of the 65 individuals, also inferior in flexion (fig. 3 ). In only nine of 65 subjects in a state of head flexion was any portion of the hyoid bone level with, or superior to, the inferior edge of the mandible. In none of these nine cases, was the entire bone observed to be superior to the inferior edge of the mandible. Thus, for the radiographs summarized in figure 2, slight differences in degree of head flexion or extension comprise an insignificant source of error.

Unlike the hyoid bones of all other specimens shown in figure 2 , a portion of the hyoid bone of the adult chimpanzee is located superior to the inferior edge of the mandible. The corpus of the hyoid bone is located at approximately the level of the gonial angle (i.e., level with the inferior edge of the mandible), while the greater horns of the hyoid bone curve posteriorly and superiorly behind the level of the posterior borders of the ascending rami of the mandible. The position of the corpus of the adult chimpanzee hyoid bone shown in figure 2 is comparable to those shown by other researchers. Swindler and Wood picture a similar position for the chimpanzee ('73:89). Analogous positions have been illustrated for the corpora of hyoid bones of the gibbon (DuBrul, '58:60), gorilla (Negus, '49:28) and orangutan (Negus, 49:27). Unfortunately, midsagittal illustrations do not permit comparison of the greater horns of hyoid bones.

It is interesting that the hyoid bone of stillborn chimpanzees is more inferiorly and anteriorly placed than that of the adult chimpanzee. At first glance, it would appear that the widely accepted principle that the larynx descends during the individual's lifespan is contradicted by the comparison. However, the apparent discrepancy is due to the fact that while the larynx may indeed descend in relation to cervical vertebrae (Jordan, '71), it does not do so relative to the mandible, the reference structure used in this paper. The change in positions of the stillborn and adult hyoid bones, noted above, is therefore probably the result of mandibular growth.

The hyoid bone of man and that of apes differ in both morphology and function. Two major morphological differences appear between the hyoid bone of a chimpanzee (or gorilla) and that of a man: a chimpanzee's is relatively larger and its corpus is markedly hollowed-out and cupped.

In both of the chimpanzees dissected, the corpus of the hyoid bone appeared as a superior continuation of the laryngeal air sacs which extend directly under the hyoid bone from the ventricle of the larynx out through the thyrohyoid membrane. Air sacs, which do not normally occur in man, are usually present in male chimpanzees. Vertical movement of the hyoid bone compresses the orifice of laryngeal air sacs (Jordan, '71:104) and thus probably participates in the mechanics of filling up and emptying these sacs.

Dissection of both chimpanzees confirms Jordan's finding that the epiglottis of the chimpanzee is higher, relative to the palate and is wider in its upper part than that of man (Jordan, '71:113). In both excised larynges, the epiglottis could be tilted to meet precisely the complex of cuneiform and corniculate cartilages, thus covering the inlet to the larynx (fig. 4). 
A

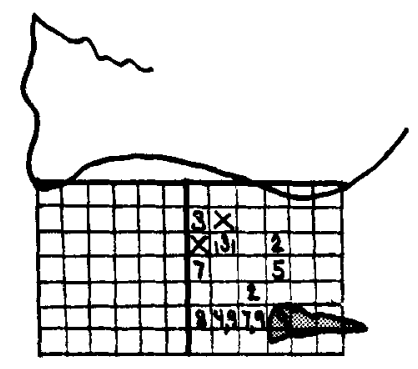

B
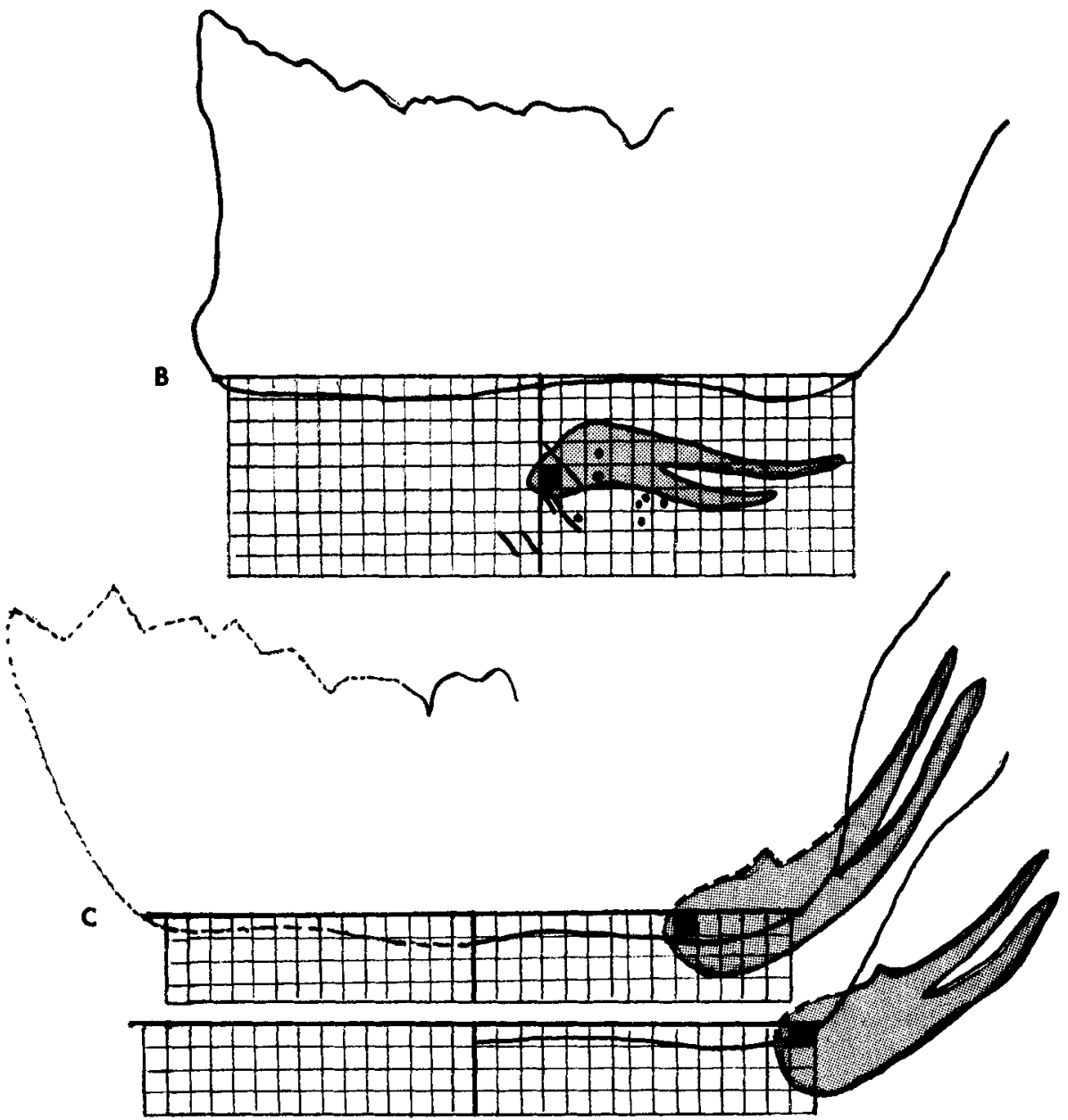

Fig. 2 Hyoid bone relative to mandible in humans and chimpanzees. Numbers and symbols indicate most superior rostral full square located within corpus of hyoid. Eight squares $=$ one inch. Hyoid bones drawn in to show method of locating most rostral square. Data summarized from radiographs. See also figure 3 and discussion in text of significance of head extension and flexion to position of hyoid. A, infant humans and stillborn chimpanzees; numbers 1-9 indicate age in months of infant humans $(n=14), x=$ stillborn chimpanzees $(n=2) . B$, adult humans; dots and solid square $=$ females $(n=8)$, oblique lines $=$ males $(n=7)$. C, adult female chimpanzee from oblique radiograph (above) and corrected to lateral view (below). 


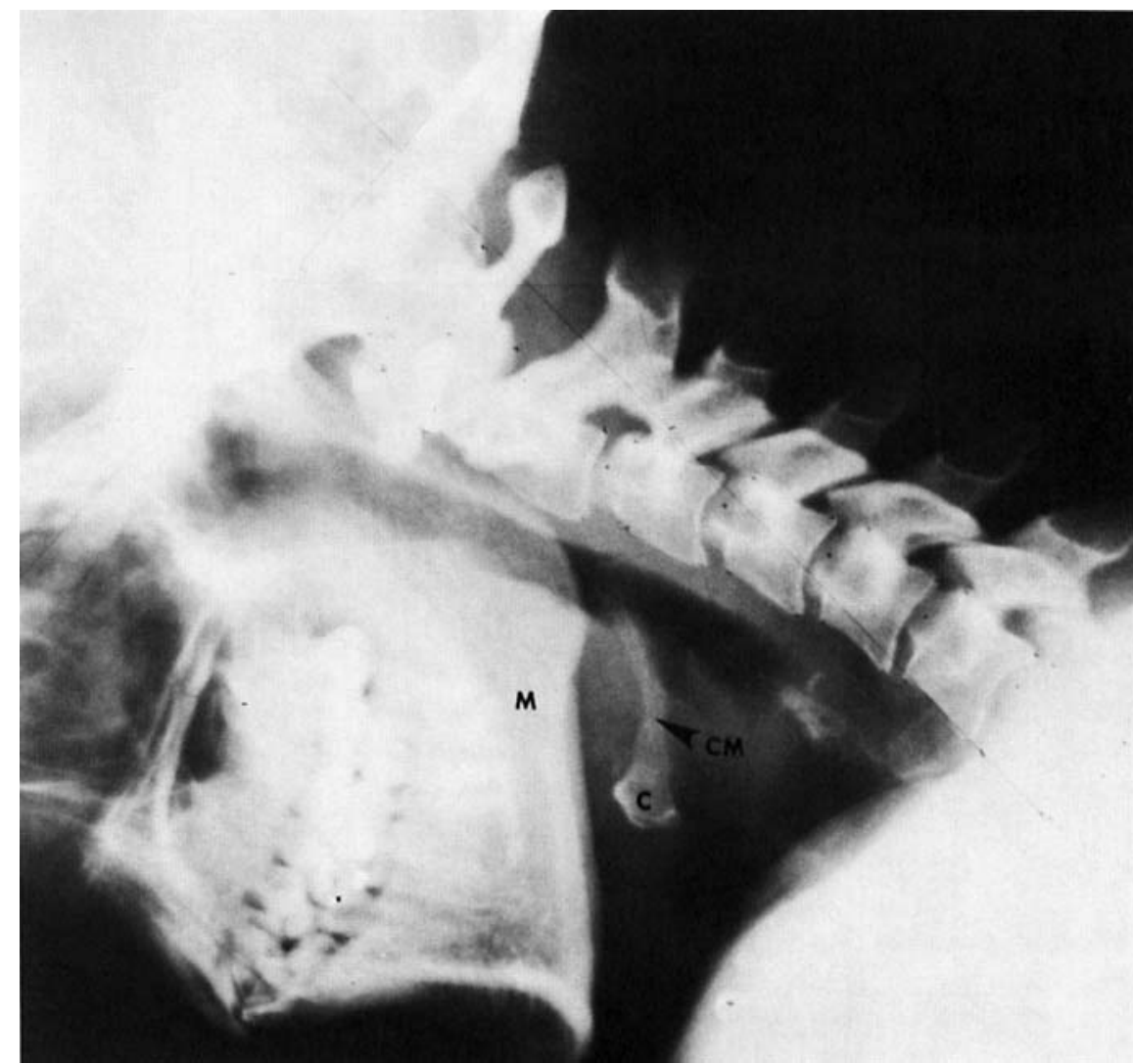

Fig. 3 Hyoid bone relative to mandible in adult female human during head flexion. Abbreviations: $M$, mandible; $C$, corpus of hyoid bone; $C M$, greater horn of hyoid.

This tilting of the epiglottis would occur in living chimpanzees as a result of pressure from the base of the tongue since the upper portion of the epiglottis of the chimpanzee is "depressed downward by the base of the tongue" (Kelemen, '69:169). This hypothesis is in need of cinemagraphic confirmation.

\section{DISCUSSION}

According to Negus ('49), the descent of the larynx in man is correlated with the evolution of erect posture. The epiglottis of man is thus separated from the soft palate, rather than approximating it as is the case in the chimpanzee. The relationship between the epiglottis and the entrance to the larynx in man is such that the epiglottis does not protect the larynx during swallowing. The entrance to the larynx must therefore be closed by other mechanisms so that food or liquid will not go "down the wrong pipe."

Swallowing in humans consists of closing the mouth and raising the tongue to the palate which forces the substance to be swallowed into the oral pharynx. The breath is held and the substance is propelled down the esophagus by the constrictor muscles of the larynx. The hyoid bone is pulled upwards and forwards by contraction of the muscles of the submandibular region. Although the base of the elevated tongue presses upon the tip of the epiglottis, the epiglottis "does not fall down over the opening of the larynx as a trap door" (Crafts, '66:567) because the strong hyoepiglottic ligament, which is pulled upwards and forwards by the mobile hyoid bone, acts as a restraint. Therefore, only 

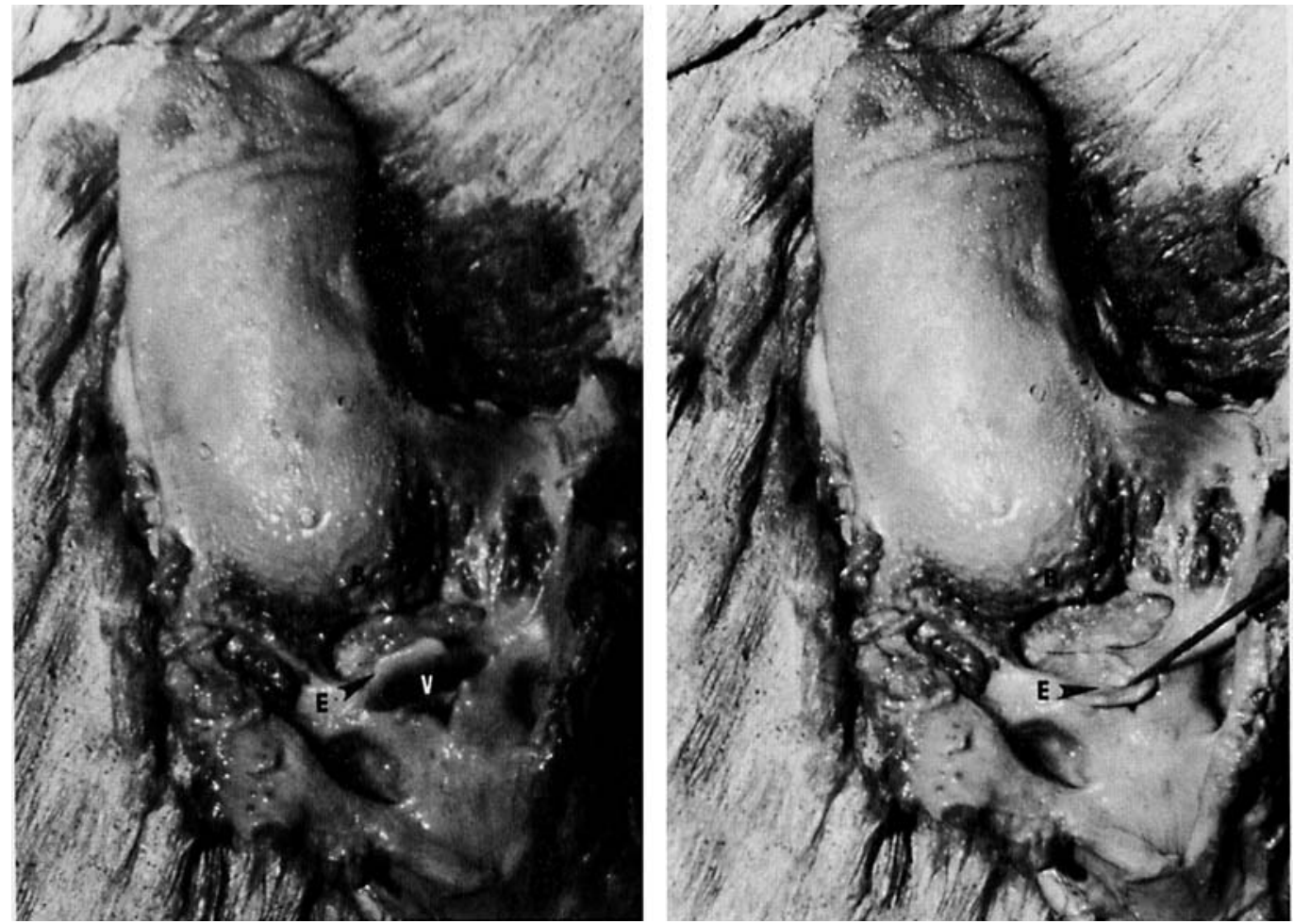

Fig. 4 Excised tongue and larynx of chimpanzee. Left, epiglottis (E) in resting position; Right, epiglottis pressed down to cover vestibule of larynx (V), B, base of tongue.

the tip of the epiglottis turns down when swallowing (Johnstone, '42:97).

Two mechanisms function to prevent a human from choking on food or liquid which is swallowed: (1) the rima glottidis, or vocal cords, close during swallowing (Negus, '49:166). (2) Fibrous aryepiglottic folds contract when an elevated hyoid bone brings the tip of the epiglottis up against the base of the downward pressed tongue via the hyoepiglottic ligament. The tips of the arytenoid cartilages and epiglottis are therefore compressed towards each other, thus causing protective puckering of the aryepiglottic folds over the vestibule of the larynx.

The chimpanzee lacks both mechanisms which protect the human larynx during swallowing. Unlike humans, the chimpanzee has a hiatus intervocalis which prohibits complete closure of the vocal cords (Kelemen, '69:182). The chimpanzee also lacks fibrous aryepiglottic folds which contribute to the protection of the human larynx (Jordan, '71:125). The fact that the epiglottis could be tilted to meet the cuneiform and corniculate cartilages in both chimpanzee cadavers suggests that the epiglottis of the chimpanzee plays a greater role in protecting the larynx during swallowing than does the epiglottis of man. That is, the base of the tongue in the swallowing chimpanzee presses on the epiglottis causing it to cover precisely the entrance to the larynx (fig. 4).

Figure 5 shows the reconstructed larynx of Neanderthal (Lieberman and Crelin, $71: 208)$. The position of the hyoid bone of Neanderthal was determined in part from its intersection with the reconstructed stylohyoid ligament and reconstructed geniohyoid muscle (' $71: 208$ ). This method of determining the position of the hyoid bone of Neanderthal is questionable since $\mathrm{La}$ Chapelle has but a small portion of the left styloid process from which to reconstruct the stylohyoid ligament. Lieberman and Crelin restored the styloid process and 
stylohyoid ligament at an angle of approximately $46^{\circ}$ from the axis of the zygomatic arch (fig. 5), while from a photograph of the actual skull, I measure an angle of $67^{\circ}$ between the line of the styloid process and zygomatic arch axis (fig. 6).

It would also be difficult to determine accurately the angle of the geniohyoid muscles from genial tubercles because geniohyoid muscles angle in an inferior, posterior direction from the symphysis of the mandible whereas genial tubercles, which are small in casts and photographs of $\mathrm{La}$ Chapelle and small and variable in man, usually do not. Accurate positioning of the hyoid bone relative to the mandible is especially important in the case of La Chapelle because one cannot locate the hyoid bone relative to the cervical vertebrae since the crucial second, third, and fourth cervical vertebrae are missing in this specimen (Straus and Cave, '57:353).

The hyoid bone of Neanderthal has been reconstructed, by Lieberman and Crelin, completely superior to the inferior edge of the mandible and its corpus approximates the vertical line which bisects the gnathiongonion distance (fig. 5 ). Hence, it has been reconstructed more anteriorly than the hyoid bone of an adult chimpanzee and more superiorly than those of newborn and adult humans and stillborn chimpanzees. Contrary to the view taken here, the following quotation implies that the larynx of La Chapelle has been reconstructed too low rather than too high (Lieberman and Crelin, '71:209):

Although the larynx was judged to be as high in position as that in Newborn and apes, it was purposely dropped to a slightly lower level to give Neanderthal every possible advantage in his ability to speak. . .

Figure 5 suggests that the reconstructed Neanderthal would be unable to swallow like a human. The anterior bellies of the digastric, which originate on the inferior surface of the mandible near the midline, would insert in their intermediate tendons (attached to the hyoid bone) above their level of origin rather than below as is normal for man. Consequently, the anterior digastrics would have a depressing rather than the normal elevating action upon the hyoid bone. Similarly, a comparison of $\mathrm{Ne}$ anderthal and adult man in figure 5 suggests that the main action of the geniohyoid muscle of Neanderthal would be that of pulling the hyoid bone forward in the act of swallowing, rathen than raising it as is normal for man. Other muscles, such as the mylohyoid, would perhaps retain the function of elevating the hyoid bone in the reconstructed Neanderthal.

The direction in which the hyoid bone moves during swallowing is determined by computing the vector sum of the actions of all the muscles acting on the hyoid bone. Although the information necessary to compute the vector sum for Neanderthal does not exist, figure 5 suggests that two of three important "elevators" of the hyoid

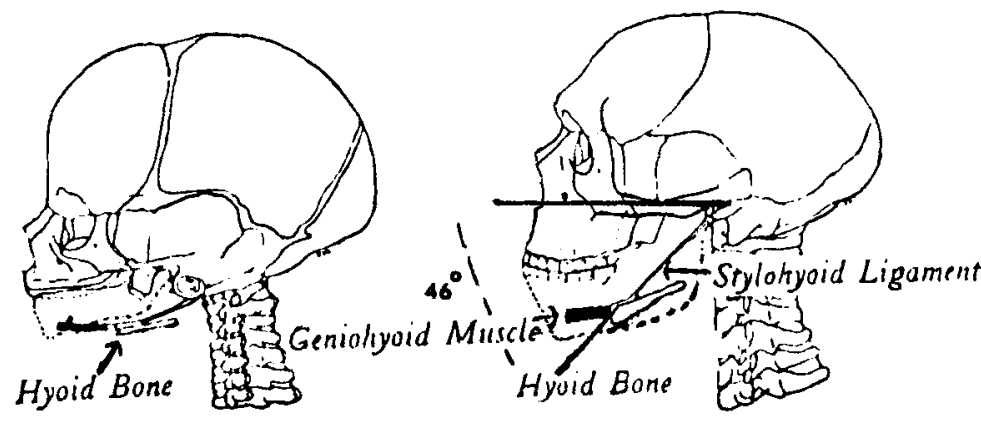

Newborn

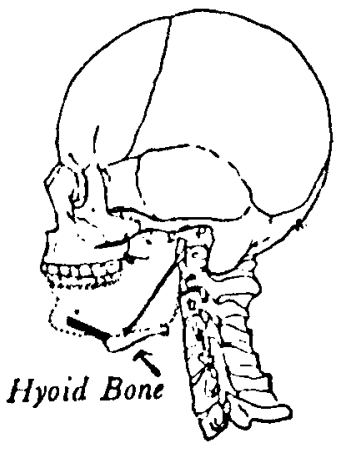

Adult Man

Fig. 5 Position of hyoid bone relative to mandible in Neanderthal, newborn and adult humans. From Lieberman and Crelin ('71), with angle between axes of stylohyoid ligament and zygomatic arch added. 


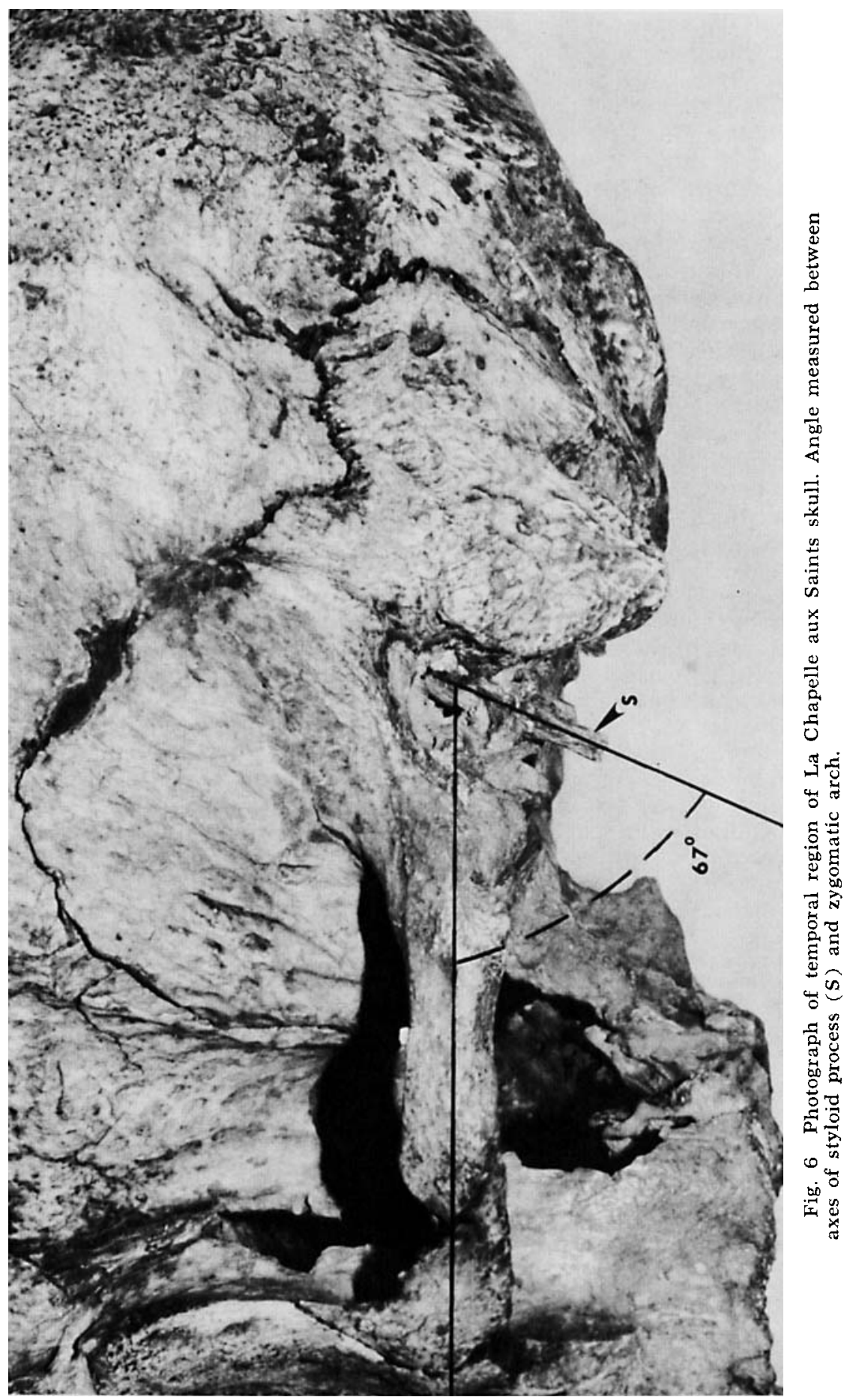


bone, as discussed above, would have actions different in Neanderthal from those in modern man. Therefore, it is doubtful that synergistic action of the muscles of the submandibular region of Neanderthal would result in an elevated hyoid bone during swallowing, and since the space above the hyoid bone of Neanderthal is crowded by muscles of the tongue (see Lieberman and Crelin, ' $71: 204$, fig. 7), it is also difficult to conceive of where the hyoid bone would elevate to. Since elevation of the hyoid bone in humans draws the epiglottis superiorly against the base of the tongue, thus bringing about contraction of the aryepiglottic folds (Johnstone, '42), it seems unlikely that the reconstructed Neanderthal would have been able to swallow like modern man.

\section{CONCLUSION}

As previously stated, separation of the epiglottis from the soft palate, which is characteristic of man but not the chimpanzee, is correlated with the evolution of erect posture (Negus, '49). Since La Chapelle represents an upright, bipedal hominid there exist "no reason (to assume that he) differed fundamentally from modern man in his cervical curvature and the carriage of his head" (Straus and Cave, '57: 356 ). Thus one would expect the oral air passage to form a right angle with the oral pharynx as is the case for man rather than to form the wider angle which characterizes the slump postured chimpanzee (Swindler and Wood, '73:89 for illustration). Any attempt to reconstruct the larynx of Neanderthal should take into account the correlation between bipedalism and descent of the larynx and should produce anatomical relations between the epiglottis, entrance to the larynx and position of the hyoid bone that permit swallowing in the human manner.

Use of the larynx of a newborn human as a model for reconstructing the larynx of a mature, erect hominid does not produce these relations for various reasons: newborn humans are not able to swallow solid food (and are known for choking, sputtering, gasping and burping even the liquid food provided them). Nor are newborn humans upright. In any event, the newborn human does not justify placement of the hyoid bone of Neanderthal superior to the inferior edge of the mandible, since the entire hyoid bones of all 14 human infants appeared below this edge.

Use of a chimpanzee model to reconstruct the larynx of an upright hominid is also inadvisable. Chimpanzees are not habitually bipedal, a fact which is reflected in the carriage of the head as well as the high position of the epiglottis relative to the soft palate. Further, both the position and morphology of the chimpanzee hyoid bone differ from those of man. Finally, the hyoid bone of the chimpanzee participates in the functioning of laryngeal air sacs, which are not present in humans.

The skeletal similarity between Neanderthal and modern man suggests that the larynx of Neanderthal should be reconstructed like that of an adult human, i.e., with a hyoid bone well below the inferior edge of the mandible, with human-like muscles of the submandibular region, and with an epiglottis which is relatively low and distant from the soft palate. Such a reconstruction would place the larynx of Neanderthal in a lower position than that pictured in figure 5 and thereby would increase the area of the supralaryngeal vocal tract and probably alter its speech functions.

\section{ACKNOWLEDGMENTS}

I would like to thank Dr. R. Stalnaker and Professor R. Snyder of the University of Michigan Highway Safety Research Institute, the former for supplying two chimpanzee cadavers and the latter for providing figure 3 as well as access to additional radiographs of adult humans. I am indebt. ed to Dr. A. Poznanski of Mott Children's Hospital, Ann Arbor, Michigan, for the opportunity to view radiographs of human infants and am also grateful to Dr. F. Jenkins, Jr. of the Museum of Comparative Zoology, Harvard University, for providing figure 1 and radiographs of stillborn chimpanzees. I thank M. Lux for her helpful reading of the manuscript and $D$. Frayer for photographing figures 4 and 6 . I am most grateful to Professor Ernst Goldschmidt of the Department of Anthropology, University of Michigan, for his considerable help and encouragement and to 
Dr. L. Radinsky of the Department of Anatomy, University of Chicago, for help in preparing this manuscript.

\section{LITERATURE CITED}

Crafts, R. C. 1966 A Textbook of Human Anatomy. The Ronald Press Company, New York.

DuBrul, E. L. 1958 Evolution of the Speech Apparatus. Charles C Thomas, Springfield.

Johnstone, A. S. 1942 A radiological study of deglutition. J. Anat., 77: 97-100.

Jordan, J. 1971 Studies on the structure of the organ of voice and vocalization in the chimpanzees. Folia Morphologica (Warsz.), 30: 97-126.

Kelemen, G. 1969 Anatomy of the larynx and the anatomical basis of vocal performance. In: Anatomy, Behavior, and Diseases of Chimpanzees. G. H. Bourne, ed. University Park Press, Baltimore, pp. 165-186.
Lieberman, P., and E. S. Crelin 1971 On the speech of Neanderthal man. Linguistic Inquiry, 2: 203-222.

Lieberman, P., E. S. Crelin and D. H. Klatt 1972 Phonetic ability and related anatomy of the newborn and adult human, Neanderthal man, and the chimpanzee. Amer. Anth., 74: 287-307.

Morris, D. H. 1974 Neanderthal speech. Linguistic Inquiry, 5: 144-150.

Negus, V. E. 1949 The Comparative Anatomy and Physiology of the Larynx. Hafner Publishing Company, New York.

Straus, W. L., and A. J. E. Cave 1957 Pathology and the posture of Neanderthal man. Quarterly Review of Biology, 32: 348-363.

Swindler, D. R., and C. D. Wood 1973 An Atlas of Primate Gross Anatomy. Baboon, Chimpanzee, and Man. University of Washington Press, Seattle. 